\title{
Los elementos objetivos y subjetivos en la definición internacional de la agresión*
}

El profesor Paul Reuter ha demostrado con una pertinente claridad los problemas teóricos y prácticos que se presentarían si la comunidad internacional consiguiese colocar la fuerza al servicio exclusivo del derecho'. Se necosiliaria una revolución y la existencia de una institución: revolución al establecer un sistema jurídico completo, en el cual las diferentes funciones internacionales estarian distribuidas y ordenadas; pero se requeriria igualmente una institución, es decir una organización internacional, la cual deberia asumir complejas tareas, tales como constatar una agresión, una falta al orden juríclico estableciclo, reprimiénclola con la ayuda de una fuerza independiente, imparcial e intemacional.

Estas ideas guias que el profesor Reuter ha diseñado, aparecen confirmaclas en he práctica internacional. Cincuenta años se ha demorado la sociedad internacional para convencerse de la utilidad de clefinir la agresión y concluir sobre el contenido intrínseco de la misma ${ }^{2}$.

Ya en cl seno de la Sociedad de las Naciones, la cuestión de la agresión había sido objeto de interesantes deloates ${ }^{3}$. Después y a raíz de una proposición de la Unión Soviética, la Asamblea General de Naciones Unidas en su quinta sesión incluyó $\mathrm{el}$ problema de la agresión entre los puntos cle examen. Curiosanente por más de veinte y dos años, la cuestión de la clelinición de la agresión siguió apareciendo en el índice de materias que la Asamblea General debía tratar en cacla sesiónt. PPero su inclusión no significaba unanimidad en

- Este estudio se realizó en el Institut du Droit de la Paix et clu Développement de la Universidad cle Nice, gracias a una invitación del Gobicrno de Finncia.

${ }^{2}$ CE. Droit International Public., J'aris, rui, 1973 (Jq edition) 473 pp. 431. 443.

"Véase Zourek (J.) Enfin une définition de l'agression., AFm, 1974., pp. 9 . 30; p. 9.10.

solıre los aspectos ya históricos de la definición de la agresión, véase el es. udio de Komarwicki (W). La définition de l'agresseur dans le droit international moderne, :can, 1949, vol. 75., pp. 1-113; igualunente Zourek, (J). La définition de l'agression et le droit international. Développements recents de la question., RCADl, 1957, vol. 92., ip. 755-860.

'En curmo al desarrollo de este problema en el marco de Naciones Uniclas, se pueden consultar los informes anuales de Raton J'. en el AFor, desrle 1968 a 197t; también el articulo de Torres Bernardez. Examen de la deffinition de 
cuanto a la utilidad de contar con una definición, pues algunos la consideraban simplemente inútil $y^{\prime}$ aun peligrosa.

No sin razón y' con algo de thumor, hichel Virally se expresaba de la definición de la agresión como de una serpiente de mar que resurge periódicamente, pero que nadie ha podido wer con claridads.

En medio de tantas oposiciones vino la transacción: en 1953 y 1956 la Asamblea General creó un Comité especial para estudiar la cuestión de la clefinición de la agresión, en el seno del cual se presentaron varios proyectos de diferénte contenido. Unos incluían aspectos exclusivamente militares, otros agregaban clementos tipificadores de

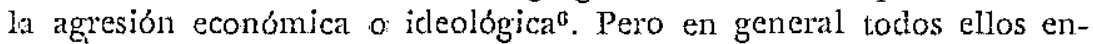
tregaban una delinición mixta combinando una cláusula general con una enumeración ejemplar de casos de agresión.

Sin embargo, y at raíz de problemas de procedimiento, los trabajos referentes a la cuestión de la agresión se suspendieron por más de once añosi. Solamente a partir de 1967, el Comité encargado de este punto decidió continuar con su labor, contando para ello con el apoyo de nuevos estaclos miembros de la organización. En las reuniones de marzo a abril de 1974, el Comité pudo presentar a la Asamblea General, un proyecto de resolución que fue aceptado por ésta en la xxix sesión. La definición adoptada -como lo señala por lo demás Pierre Raton- "no satisface plenamente a nadie, pues ella es el resultado de un compromiso y presenta deficiencias" 8 .

La resolución que contiene la definición de la agresión es un compromiso entre los autores de tres proyectos que se presentaron durante 1969. En efecto, el comité especial reunido en Nueva York entre el 124 de febrero y el 3 de abril de 1969, conoció tres proyectos de definición de la agresión: el primero, presentado por la Unión Soviética; el segundo, llamado proyeoto de 13 países (Ohipre, Colombia, Ecua-

I'agression., AFDr, 1965., pp. 528-545. Sobre aspectos puramente juridicos véase Chaumont (Ch.) Explication juridique d'une definition de l'agression., AFDr, 1956; pP. 52I-529; del mismo autor. La définition de l'agression en 1970-1971; en Miscellanca W. J. Ganshof Van der Meersch., tomo I. Bruxelles. Et.Emile Bruylant., 1972., pp. I15-129; por último Ramband (l'.). La définition de l'agression par l'oNu, RGDP., 1976; pp. 335-\$81.

${ }^{5}$ Cf. L'Organisation Mondiale, Paris, Amand Colin, 1972. 587 pp. 447.

'Véase Chaumont (Ch.). Explication juridique d'une définition de l'agression... op. cit., p. .593

TEn efecto, en 1955, 22 Estados fueron admitidos en la Orgnnización de Naciones Unidas, situatión que condujo a la Asamblea General a solicitar de ellos sus opiniones frente a la elefinición de la agresión, encargando a la mesa de la Asamblea el estudio de las respuestas. Este proceclimiento significó un retardo, sir perjuicio de que la composición de la mesa no favoreció una agilidad a raíz de su oposición a definir la agresión.

${ }^{8}$ Cf. Travaux de la Commission Juridique de l'Assemblec Générale des Nations Unies (xxIX Session), AFvr, 1974, pp. 521-552., pp. 528-529 
dor, España, Ghana, Guyana, Haití, Irán, Madagascar, México; Uganda, Uruguay y Checoslovaquia); y por último aquel presentado por seis Estados Occidentales (Australia, Canadá, Estados Unidos, Italia, Japón e Inglaterra) ${ }^{2}$.

Las principales deficiencias dicen relación al principio de anterioridad que, según ciertos comentadores, podria ser exclnido por la acción del Consejo de Seguridad alegando la inexistencia de una intención agresiva ${ }^{10}$.

Esta idea de "intención agresiva" nos introduce ya en el corazón mismo de este estudio. La finalidad asignada a éste consiste en analizar el papel que juegan los elementos objetivos y subjetivos en la definición de la agresión tal cual dia sido adoptada por la Asamblea General11.

La óptica escogida no significa que neguemos que el concepto mismo de agresión supone la reunión o la combinación de los clementos objetivos y subjetivos. Sin embargo, la preponderancia que se le dé a uno u otro va a implicar consecuencias jurídicas importantes. Por otra parte, el predominio de uno de los elementos depende de una concepción política más general, que dice relación entre otras cosas al papel que se le otorgai a Naciones Unidas, particularmente al Consejo de Seguridacl, en los conflictos internacionales.

Estudiados esos aspectos descle un punto de vista teórico-doctrinal, aparece necesario analizar después cómo han sido combinados en la práctica ambos elementos. La definición de la agresión según la resolución 3314 ( $\mathrm{x} \times \mathrm{x}$ ), nos servirá en clicho análisis.

Pero si el concepto de agresión se obtiene sumando o combinando los elementos objetivos y subjetivos, parece importante señalar en qué consiste cada uno de ellos. Objetivamente, la agresión es una falta al derecho internacional que resulta de la realización de ciertos hedhos prohibidos; subjetivamente la agresión se va a caracterizar por Ia finalidad o motivación, situación que permitirá una interpretación correcta de los hechos acaecidos a fin de identificarla indiscutiblemente.

Ambos elementos son complementarios y deben encontrarse cuando se persigue definir la agresión ${ }^{12}$. Sin embargo, la mayor im${ }^{\circ}$ Citado por Raton (P.)., Travaux de la Commission Juridique de l'Assem. blée Généralc des Nations Unies (xxiy Session), AFDI 1969., pp. 402.457., p. 449.

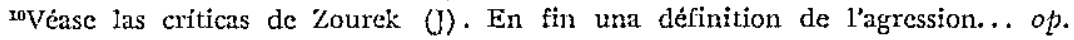
cit., pp. 25-27. Sobre este punto nosotros volveremos más adelante.

"La Asamblea General adoptó el proyecto de resolución (A/C.6/L. 993), por consenso, no obstante que ciertas delegaciones hayan estimado su disconformidad con dicho procedimiento. Ante la demanda del representante chileno de que figurara dicho país en la lista de autores, diversos problemas se produjeron frente a la negativa por parte del Presidente de aceptar ese copatrocinio sin consultar a los autores originarios.

1Cf. Ramband (P.). La définition de l'agression par l'owu, R, G. DIP; op; cit., p. 864 . 
portancia que se le otorgue a uno respecto del otro (hecho ilícito sobre intención agresiva o viceversa), provocará ciertos problemas que penetran en el campo gencral de la polítioa y del derecho.

I. El predominio de uno de los elementos apumita a un problema general de la politica y del derecho.

Desde un punto de vistai político la mayor o menor importancia que se le asigne a los elementos objetivos y subjetivos desembocará necesariamente en un nuevo análisis de las condiciones de la agresión como de su constatación.

En cambio, la preferencia de uno de Jos elementos ya individualizados, nos conclucirá jurídicamente a problemas relacionados con el fundamento de la responsabilidad y con la naturaleza o extensión de la interpretación.

\section{A. Las oposiciones politicas y sus consecuencias.}

Las consecuencias de lo que themos llamado "oposiciones políticas" aparecen a dos niveles: en primer lugar, a nivel de las condiciones de la agresión: segundo, en lo que respecta a la constatación de la misma.

Es un thecho que todo aquel que utiliza la fuerza, entendida en su sentido amplio, busca justificarla. Esta necesidad aparece particularmente resentida cuando la fuerza supone la utilización de medios militares. No en vano el Secretario General Breynev a raíz de los hechos ocurridos en Checoslovaquia, expresaba que la "soberanía está subordinada al respecto por el E⿱Esstado socialista de la leyes Generales de Ia edificación socialista como también a los intereses y a la seguridad de toda la comunidad socialista"13.

'Aún en materias internacionales económicas, cuya originalidad proviene especialmente, porque estamos en un campo en clonde el clerecho se toca con el antiderecho o la política con un germen de derecho ${ }^{14}$, los Estados, las Organizaciones Internacionales o las personas privadas que thacen uso cle la fuerza, véase el Boycott por ejemplo, buscan compatibilizarlo al menos con el derecho internacional general15.

${ }^{23}$ Citado por Fischer (G.)., Quelques problemes juridiques découlant de l'affaire Tchecoslovaque., AFDI, 1968. pp. 15-42; p. 23 Discurso de Varsovia, 12 de noviembre 1968.

${ }^{1 * A}$ este propósito se puede consultar el artículo de Weil $\langle\mathbf{P}$.$\rangle . Le droit in-$ ternational Economique Mythe ou Realitë? en Aspects du droit international economique, Paris, Editions Pédone; p. 972, pp. 3-34.

rvéase el estudio de Lucchini (L)., Le Boycottage en Ia obra Aspects du droit international economique... op. cit. pp. 67-101 
La justificación persigue entonces extraer del thecho el aspecto de ilicitud, dejánclolo desprovisto de toda colonación penal. Uno de los meclios más socorridos de justificación dice relación a la finalidad o motivación que indujo a un determinado sujeto a actuar. No bastaría la simple realización de hechos materiales, habria que investigar cuáles han sido los motivos.

La búsqueda del elemento teleológico, de la intención agresiva, introduce, pues, variantes en el campo de las condiciones de la agresión y permite efectuar distinciones.

Habrá agresión cuanclo se reúnan el hecho material y la intención agresiva. Sin embargo, la transformación de la intención agresiva en "intención en defensa del derecho", por ejemplo, nos conduciría a una atilización legal de la fuerza; en resumen, nos conduciría a una no-agresión.

Estas ideas algo teóricas deben relacionarse con el medio internacional, es decir con la política mudial, en donde prevalecen según la fórmula de Stanley Hoffman "el conflicto sobre la cooperación" y aun más en donde el conflicto tiende a clesembocar en la violencia.

El problema se complica frente al hecho de que en ausencia de un poder temporal superior a las diversas unidades estatales en concurrencia, éstas conservan la libertad de recurrir a la fuerza para alcanzar sus objetivos.

Cada Estado podrá considerar que recurrir a la fuerza para alcanzar objetivos que le son propios, véase intereses vitales, es una presunción legal de inexistencia de intención agresiva.

Es cierto que estos raciocinios olvidan las múltiples combinaciones de fuerza cque en el tiempo $y$ en el espacio pueden resultar de las relaciones entre Estados. Estos últimos tejen a su alrededor una serie de relaciones persiguiendo acrecentar su influencia, defenderse contra una eventual agresión o simplemente dotarse a través del comercio de los medios necesarios para su existencia.

Sin embargo, cualquiera sean las posibles combinaciones de fuerza o "constellations diplomatiques" como las llama Raymond Aron, el problema de la agresión sigue en pie, esta vez a nivel de su constatación.

Sabemos que a partir clel ya clásico principio del equilibrio político, diferentes sistemas internacionales han sido diseñados. Olvide. mos por el momento los modelos históricos, tales como aquel fundaclo sobre Ia "civitas christiana" o aquel otro implementado con tanta frialdad por Richelieu o, por último, el expuesto sumariamente por Hume en la obra significativa por su título, "On the balance of power".

Los principales modelos actuales, el sistema internacional bipolar y los sistemas multipolar o pluripolar no nos solucionan definitivamente el problema de la agresión. 
En un sistema bipolar, la luerza (o agresión) puede ser utilizada para prevenir un aumento de poderío por parte del otro Supergrande o de su bloque. Pero también ella puede ser usada para mantener la cohesión del campo en cloncle el SupertGrande ejerce influencia. El surgimiento del arma atómica ha clisuadiclo a las dos grandes potencias de lanzarse en una guerra total, los ha incitado a la moderación, recomendándoles no desconocerse sus intereses vitales.

En resumen, en la era nuclear es imposible qque la fuerza sea ejèrcida de una Superpotencia contra la otra directamente. Lo que si ha ocurrido en que una Superpotencia tha ejercido la fuerza para prevenir un aumento significativo de pocler por parte del bloque contrario, alegando que de alcanzarse dicho aumento estaríamos frente a un desconocimiento de los intereses vitales de la primera. Bajo esta perspectiva podríamos anatizar la crisis de Cuba en 1962.

Un enfrentamiento tan agudo como aqquel que ocurrió en Cuba clemuestra la imposibilidad por parte de la comunidad internacional cle determinar al agresor, los intereses en juego van más allá, desconocen los clispositivos juríclicos e institucionales de que se ha dotado la Organización Mundial para solucionar conflictos medios o menores.

¡Pero la fuerza puede ser utilizadla en un sistema bipolar con un segundo objetivo: mantener la cohesión del campo de influencia de una Gran Potencia. ILos hechos ocurridos en República Dominicana y en Checoslovaquia han sido ya analizados descle múltiples ángulos, por lo cual no volveremos al detalle de ellos. Sí, nos interesa señalar que nuevamente el aparato institucional tha demostrado su incapacidad para introducirse en el coto de las grandes potencias. Cualquiera proposición tencliente a calificar una determinada acción como constitutiva de agresión se habría topado con el derecho de veto que asegura, entre otras cosas, el equilibrio entre quienes lo detienen.

Veamos lo que ocurre en un sistema intemacional multipolar o pluripolar. Una de las caractenísticas estructurales de este sistema es la existencia de una pluraliclad de actores que disponen de recursos semejantes (capacidad, medios, fuerza). El poderio no es igual, pero la desigualdad de medios no crea unat disparidad de naturaleza. $\mathbb{E} 1$ "juego" internacional se funclamenta sobre una relación de fuerzas moderadas por el equilibrio de una pluralidad de Estados rivales o competitivos.

Todos los autores que se than preocupado de analizar este sistema multipolar o pluripolar concuerdan en reconocerle en principio una mayor capacidad para limitar la agresión. Así, un sistema "homogéneo" en el pensamiento de Ralymond Aron o un sistema "moderado" en la concepción de Stanley Hoffmann favorecerian una limitación de la 
violencia. Sin embargo, tal afirmación no significa que los actores principales hayan renunciado a utilizar medios no pacíficos. Lo que sucede es rque el relativo equilibrio existente incita a los actores a fijarse objetivos menos ambiciosos que pretenden alcanzar por medios moderados, algunas veces, por medios pacíficos, en otras oportunidades a través de guerras limitadas. Haciendo abstracción del momento thistórico, podríamos analizar bajo esta perspectiva la intervención francoibritánica en Egipto (1956). Hemos escogido este caso porque ambos paises: Trancia e Inglaterra, son potencias medias que cuentan desde un punto de vista institucional con todo el arsenal de privilegio que les otorga su calidad de miembros permanentes del consejo de Seguridad de Naciones Unidas. Aun frente al rechazo de la intervención por parte de Estados Unidos y de la Unión Soviética, tropas franco-británicas penetraron no obstante en territorio egipcio en octubre de 1956, alegando que con ello salvaguardaban la libertad de paso a través del Canal de Suez.

La calidad de los Estados que intenwinieron y los motivos on que fundamentaron la utilización de la fucrza, condujeron a paralizar la iComunidad Internacional en cuanto a una posible constatación de la agresión.

Analizado desde una perspectiva política, el predominio de uno de los elementos constitutivos de la agresión, conviene por ahora referirse a las incidencias junidicas.

\section{B. La quevella juridica y sus incidencias.}

La preponderancia del elemento objetivo o del elemento subjetivo en la definición cle la agresión nos va a conducir en una dimensión estrictamente jurídica, a una serie de problemas que se relacionan con el fundamento de la responsabilidad internacional y' que afectan de alguna manera a la naturaleza de la interpretación.

(Poner el racento en el elemento objetivo, realización del hecho prohibido, o hacer hincapié en su finalidad o motivación, elemento subjetivo, ilustra ya una controversia más general.

¡Cuál es el fundamento de la responsabilidad internacional? ¿E1 simple incumplimiento? ¿La culpa o intención de dañar? La controversia es clásica y ha dividido a la jurisprudencia y a la doctrina.

En efecto, un sector importante de esta última se ha preguntado si cl simple incumplimiento bastaría para fundar la responsabilidad internacional del Estado. Conjuntamente con el incumplimiento por medio de un acto o de una omisión (hecho ilícito), habría que considerar la posibilidad de agregar la intención de dañar. Como lo señala el profesor !Quoc iDinh - - "la teoría de la falta en derecho internacional no se ha establecido bajo la sola influencia del régimen 
general de ia responsabilidad en derecho interno"10. En efecto, en materias de daños causados a sujetos o intereses extranjeros, la jurisprudencia internacional tiende a fundar la responsabilidad del Estado en cuestión a partir cle omisiones o de negligencias de las autoridades nacionales competentes. In el asunto del estrecho de Corfú, entre Albania e Inglaterra, por cjemplo ${ }^{2 T}$, este último país afirmaba entre sus conclusiones 'que Albania era responsable directa o indirectamente del minaje del estrecho. Aun cuando la prueba de que Albania hubiera participado directa o indirectamente a este tipo de operaciones no fue demostrada por Unglaterra, la Corte concluyó que $\mathrm{Al}$ bania estaba al menos en conocimiento de dicho hecho. Pero curiosamente el Tribunal no fundamentó la responsabilidad de Albania en la viII convención de la Haya como lo pretendía Inglaterra sino, más bien en la obligación 'que pesa sobre todo Estado "de ne pas laisser utiliser son territoire aux fins d'actes contraires aux droits d'autres Etats"18. Para la Corte esta obligación proviene de cicrtos principios generales o consideraciones elementales de humanidad que tienen mayor wigencia en tiempos de paz que en tiempos de guerra. Albania era, pues, responsable por actos de omisión:

En cambio, la jurisprudenciat internacional ha descartado la responsabilidad de un Estado cuando éste ha demostrado que ha hecho prueba de toda la diligencia, véase vigilancia, necesaria ${ }^{19}$. La irresponsabilidad se mantiene aun cuando el Estado no haya podido impedir la realización clel claño.

Si la jurisprudencia ha descartado la responsabilidad de un Estado aun cuando se than producido daños paxa la víctima, ello significa que las normas internacionales $\mathrm{cn}$ materia de vigilancia de un territorio, por ejemplo, contienen más bien obligaciones de comportamiento que obligaciones cle resultado"0.

Estas ideas y la acogida tque le otorgaria: la jurisprudencia, han conducido a una parte de la doctrina a una discutible generalización. Si el juez o árbitro han determinado responsabilidad, no lo han hecho consiclerando el resultado (el daño), pues en algunas casos su existencia tha sido considerada no pertinente, sino más bien fundándose en una falta concreta manifestada en los heohos de la causa a través de omisiones o simplemente a traves de vigilancias insuficientes.

${ }^{10}$ Cf. Droit international public, Paris., LGDJ, 1975, 808 pp. p. 682.

${ }^{17 T r e s}$ fallos emitió ia corte en este asunto. El primero sobre su competencia 25 de marzo de 1948; el seguntlo sobre el fondo 9 de abril de $1949 \%$ por último, el fallo de 15 de diciembre de 1949 , c11 que fija el monto de reparaciones.

${ }^{18} \mathrm{Cf}$. Fallo de 9 cle abril de 1949.

"Lil jurisprudencia utiliza la expresión "due diligence".

ovéase la jurisprudencia en el Asunto de la Isla de Palmas, árbitro Max Hulser. Sobre esta clasificación se pucde consultar la obra de Reuter (P), Droil International public... op. cit., p. 20, en donde dicho autor considera este tipo de obligaciones atenuadas o inciertas 
Trente a esta controverisia y a propósito del funclamento cle la responsabilidad, una serie de consideraciones es necesario efectuar. En primer lugar, no aparece justificado generalizar el campo de la responsabilidad en donde la falta puede tener un lugar. Todos los casos a que se thace referencia dicen relación a normas que contienen obligaciones de comportamiento (vigilancia de un territorio por ejemplo), es decir que apuntan a casos minoritarios on la teoria general de la responsabilidad del Isstado. La propia jurisprudencia no tha extendido por lo demás dichos raciocinios a otros dominios de la responsabilidiad.

Un segundo punto que sí quisiéramos señalar dice relación a unal cierta evolución que parece desprenderse de la jurisprudencia internacional. Si bien lo "ilicito" continúa constituido por una violación de una obligación internacionalan, no es menos cierto que la jurisprudencia internacional há clebicio recurrir a la introducción de ciertos elementos subjetivos para determinar oasos de responsabiliclad. Pero esta introducción ha siclo limitada, tal cual to señala el profesor Reuter, a dos grandes thipótesis ${ }^{2 x}$. LLa primera, dice bien relación a las obligaciones de comportamiento. Sin embargo, y curiosamente, la jurisprudencia clemuestra con claridad su apajo en escudriñar Ja intención agresima cuanclo quicre concluir que clla no existe en el caso de especie ${ }^{23}$; en cambio, frente a una manifiestal meilintención, los árbitros se contentan en cliscutir los elementos externos de la actitud. La segunda hipótesis, en donde la intención ha siclo consicleracla por la jurisprudencia internacional, se refiere al caso en el cual el hecho ilicito dirigiclo voluntariamente contra lia victima alcanza directamente a un tercero y secundariamente a la víctima elegida; ante esta situación el sujeto dañado o perjudicado indirecta o secundariamente puede solicitar reparación. Esta es una excepción a la regla generai24.

rUná posición particularmente clara en materia del fundamento de la responsabilidad internacional se cncuentrạ en la decisión adoptada por la comisión Irancoitaliana de conciliación el 31 de octubre de 1951 en el asunto Mossé. La comisión declaró que era impertinente detenerse a analizar si los agentes públicos italianos habían actuado con mala intención o de buena fe, pues esis circunstancias no influain en la admisión de la responsabilidad internacional.

$=$ Cf. Droit International public... op. cit. p; 180;

mVéase el asunto de James Pugh ante la Comisión arbitral entre Inglaterra $y$ Panamá; R. S. A. vol. I, p: 1416 , en donde el árbitro concluye que los policias de janamá "habian.hecho un uso razonable de su competencia $y$ de sus poderes, "Sobre el particular véase la obra de Queneudec (J. P.), La responsabilité internationale de l'Etat pour les fautes personnelles de ses agents. París LGDJ, 1966,275 pp. 241-246.

? al respecto Brigitte Bollecker - Stern en su obra "Le prejudice dans la theoric de la responsabilité internationale, Parfs, Exlitions A. Pedone, 1973, 382 págs., cita un extracto- de una decisión arbitral: "Governments like individuais are responsible only for the proximate and natural consequences of their acts... 
Pero insistir sobre los elementos materiales en una definición internacional de la agresión, que suponclua necesariamente una enumeración de thechos ilicitos, reduce en definitiva la competencia del órgano encargado de interpretar, limitándolo particularmente frente a una justilicación posterior de un eventual agresor.

Por el contrario, privilegiar los móviles que condujeron a un sujeto a realizar ciertos hechos, aparentemente ilícitos, nos conducirá probablcmente a reintroducir los riesgos de un juicio político en la determinación de la agresión. La scgunda incidencia dice relación, entonces, con toda una problemálica que afecta directamente a la interpretación.

No obstante ello, como muy bien lo ha señalado por lo demás Serger Sur ${ }^{25}$, existe en derecho internacional una imposibilidad de caracterizar la interpretación por medio de un elemento específicamente juridico. Es cierto que en el seno de la Comisión de Derecho Internacional de Naciones Unidas, la interpretación fue definida en especial por Sir Humphrey Waldock, como un proceso juríclico dotaclo de métodos particulares ${ }^{20}$. ISin embargo, la relativa unanimidad conseguida al respecto se debió en gran parte a que la comisión consicteraba la interpretación al interior de un campo particular clel derecho internacional; aquel del deredho, de los aratados.

El mérito del análisis de la Comisión es de haber escudriñado parcialmente algunos problemas referentes a la naturaleza de la interpretacion; las deficiencias dicen relación al hecho de haber limitado sus refiexiones a un campo particular, cuando la interpretación tiene por misión lijar o determinar la significación juridica de todo comportamiento de sujetos de derecho ${ }^{\mathbf{*}}$. Bajo esta perspectiva $y$ meditanclo el papel que se le hat asignado es que debemos concluir que un análisis de su naturale\%a supone precisamente extraer el proceso interpretativo del estricto derecho positivo, admitiendo la intervención de un elemento político 28 . Si tal es el correcto camino a seguir, nuestra tarea, tencliente a medir las incidencias de los elementos constitutiyos de la agresión en relación a la interpretación, se complica enomemente.

in the assence of evidence of deliberate intention to injure". CE. Dix., RsA, Vol $1 \mathrm{x}$, p. 121.

$=$ Cf. L'interpretation en droit international public., Parls, LGDJ, 1974, 149 pp. pp.70-81.

${ }^{20 V}$ Céase el in y N informe en ACDr, 1964, vol. 1t., pp. 53.67 y ACDI, 1966, vol. I., pp. 56-112, respectivamente.

${ }^{\mathrm{C}} \mathrm{Cf}$. De Visscher (Cl.), Problemes d'interpretation judiciaire en droit intemational public, Paris, Editions A. Pedone, 1963, p. 9.

zVease Sur (S.), L'interpretation en droit international Public... op. cit. p. 82: 
En efecto, no basta afirmar que la mayor o menor libertad que posee el intérprete nos conducirá inevitablemente a una interpretación más política en el primer caso, más bien juridica en el segundo. Como ya lo señaló Kelsen, la interpretación depende del poder discrecional de que está dotado el intérpretc, situándose, en consecuencia, fuera de la esfera del derecho it lo menas en forma parcial\$2. Estas icleas llevan a Kelsen a rechazar el concepto de "interpretación verclaclera": a partir de una norma o situación determinada, cliferentes interpretaciones son posibles extracr, todas igualmente váliclas, teniendo en cuenta la inevitable imprecisión de sus términos. Entre una pluralidad de interpretaciones la elección no es juriclica, ella es politica.

La aceptación del elemento político no debe, por otra parte, conclucimos a un rechazo total clel carácter jurídico de la interpretación, negándole toda reglamentación ${ }^{30}$. Lo que sí conviene tener presente es que la interpretación supone una relación estrecha entre la política y el derecho, que se manifiesta ibajo múltiples formas o modalidades y en especial si se consideran las consecuencias que pueden desprenderse de tal o cual interpretación.

Nuevamente en materia de interpretación es necesario combinar los elementos objetivos con los subjetivos, el texto con la intención de las partes, única forma de impeclir que a pretexto de consultar la intención se legalicen actos contrarios al derecho. La experiencia que nos ofrece el proceso interpretativo es válicla también en cl campo cle la delinición de la agresión.

\section{La combinación de los elementos en la definición de la agresión (Resolución $331 \%-\mathrm{x} \times \mathrm{x}$ ).}

Deciamos en la primera parte de este estudlio que como toda infracción, el concepto de agresión debía reunir tanto el elemento objetivo como el subjetivo. Ambos, Iejos de ser contradictorios, eran complementarios. [Hemos estudiado ya las consecuencias que la preponderancia de uno respecto del otro, podía acarrear, desde un punto de vista doctrinario, en el campo de la política y del derecho.

Nios corresponde en esta oportunidad descender al "terreno práctico" y ver en el texto adoptado por la Asamblea General de Naciones Unidas cómo se combinan ambos elementos. Sin embargo, ello requiere previamente de un análisis algo cronológico, tendiente a esbozar o dibujar aquel proceso que introdujo progresivamente el elemento subjetivo.

${ }^{2}$ Cf. Théorie Pure du Droit, Paris, Dallos, 1962, 196 pp. pp. 453-463.

${ }^{\circ 0} \mathrm{Cf}$. Sur (S), L'interpretation en droit international public..., op. cit., p. 84. 
A. El proceso de introducción clel elemento subjetivo.

Creemos necesario en primer lugar justificar la ausencia de referencia al elemento objetivo. Ella se comprende perfectamente pues ningún Estado ha negado la necesidad de contar con una enumeración de hechos ilícitos, precedida de una definición general. Todos los proyectos de base ${ }^{31}$ son concordantes en adoptar una definición mixta. Sin embargo, no ocurre lo mismo cuando se pretende introducir y por lo tanto considerar los móviles que llevaron a un sujeto a realizar ciertos hechos, haciendo depender en mayor o menor proporción la calificación final clel agresor de la existencia cle una intención agresiva.

La introducción del elemento subjetivo se debe especialmente al proyecto presentado por los seis países occidentales ${ }^{32}$, durante la reunión del Comité Especial para la definición de la agresión que se reunió en Nueva York, entre el 24 de febrero y el 3 de abril de 1969. Hasta esa fecha didhos paises eran contrarios, por lo general, a una definición de la agresión. Lo hicicron porque ninguno de los otros proyectas los satisfacía, en particular frente al interés que los Estados occidentales demostraban en preservar los poderes discrecionales que posee el Consejo de Seguriclad en virtud del artículo 39 de la Karta cle Naciones [Unidas ${ }^{33}$.

Presentado las tres proyectos, el Comité debió constatar que los puntos sobre los cuales existía acuerdo exan los mínimos. Los miembros del Comice aceptaban que la clefinición de la agresión debía ser compatible con la Carta de Naciones Uniclas y encontrar en ella su fundamento. Por el contrario, existian infinitas divengencias sobre todo el resto: desdc la extensión que se le debia otorgar a una posible definición hasta la oportunidad de estudiar en dicha ocasión una institución en intima relación, como era el caso de la legitima defensa.

Ante tai situación, era perfectamente comprensible que si la Unión Soviética consicleralba el principio de la anterioridad como funda. mental (el empleo de la fuerza por parte de un Estado, actuando éste primero, ser una prueba suficiente cle agresión, los Estados Unidos, por su parte, otorgaban enorme importancia a la intención existente en el agresor para poder caracterizar el hecho como internacionalmente ilícito.

${ }^{3} \mathrm{CE}$. Proyectos de la URss, de los 13 paises del Tercer Mundo y el Proyecto te los 6 passes occidentales. Todos han sido individualizados en la primera parte de este artículo.

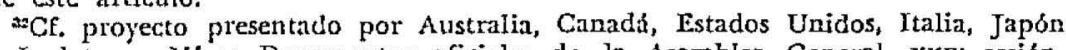
c Inglaterra. Vérse Documentos oficiales de la Asamblea Gencral, xxry sesión, A/7260,

${ }^{33} \mathrm{CE}$. la opinióll de Raton (P.). Travoux de la Commission juridique de L'Asscmblée Générale des Nations Unies, AFur, 1969, pp. 402-457; p. 450. 
No obstante tantos clesacuerclos, la Sexta Comisión de la Asamblea General pudo convocar a una nueva reunión al Comité Especial, que tuvo Iugar durante 1970 , entre el 113 de julio $y$ el 14 de agosto.

En clícha ocasión Estados Unidos, apoyaclo esta vez expresamente por Inglaterra ${ }^{34}$, thizo de la intención agresiva un elemento indispensable en la definición de la agresión. Una seric de criticas suscitó la proposición norteamerioana que, para el solo efecto de este estudio, hemos clasificado en cinco grupos.

La Unión Soviética adelantó una posición matizada, como la califica Pierre Raton ${ }^{35}$, al estimar que una agresión es siempre premeditada y que los progresos cientílicos harán muy difícil disimular sus intenciones al Estado agresor ${ }^{30}$.

México y Hungría consideraron que la intención agresiva era una noción demasiado subjetiva para ser tomada en cuenta:t.

Francia, en cambio, estimó que el criterio de intención cra incompatible con el principio de anterioridad y que no podían ser considerados y menos colocados en un mismo plano en la definición de la agresión. ILa falta de intención agresiva-agregaba el Representante francés--, no podnía significar inculpabilidacl del Estado que hubiese perpetrado primeramente un acto de agresión ${ }^{38}$.

Por su parte, Austria estimaba que la aplicación de la noción de intención agresiva podría alterar el paso de la prueba, recayendo éste en la víctima ${ }^{39}$.

Por viltimo, Chipre manifestó su oposición a introducir el elemento subjetivo alegando que su aceptación nos llevaría a la teoria medieval cle guerra justas e injustas. ${ }^{40}$

Si tales fueron los principales comentarios que el concepto de intención agresiva provocó en el seno del Comité Especial, conviene analizar además la posición doctrinal al respecto. Aprovecharemos un estuclio del profesor Chamont, que abarca precisamente ese periodo 41 .

Para Chaumont la oposición cntre los partidarios de una concepción objetiva $y^{\prime}$ aquellos que sostienen una concepción finalista es radicalmente incompatible. En la primera, el empleo nacional de la fuerza armada no es válido que en caso de legitima: defensa; en la regunda, existen otros casos, pues la concepción se inspin de la idea

${ }^{36} \mathrm{CE}$. las opiniones del representante de Inglaterra, Frecland en In 1208 sesión. ${ }^{*}$ Cf. Travaux de la Commission Juridique... op cit., «m, 1970; p; 536;

${ }^{\infty}$ Opiniones de Kolesnik, representante de la ukss, 1200 sesión.

${ }^{e r}$ Cf. las opiniones de los representantes de dichos países en las scsiones 1203 y 1209, respectivamente.

${ }^{*} \mathrm{Cf}$. opiniones del Sr. de Lacharriere, 1204 sesión.

"Ideas expresadas en la 1208 sesión.

róáce las expresiones del Sr. Rossides en 1209 sesión.

${ }^{-C}$ C. La definition de l'agression en 1970-1971 en Miscellanea W. J. Ganshof Van der Nreersch... op. cit. Pp. A19-121; 
que el fin justifica los medios y que todo aquello que no está prohi. bido está permiticlo"i2.

A pesar de estos conceptos generales, el profesor Chaumont se siente obligado a constatar que ha existido un cierto acercamiento entre occiclentales y soviéticos. Si los primeros han aceptaclo que se mencione el criterio de anterioridad, los segundos han hecho algo semejante con el criterio de finalidacl.

Las razones de este acercamiento, en especial frente a la acepoación del papel que jugaría la intención en lat definición de la agresión, se deberian a Ios hechos ocurriclos en Checoslovaquia, que habrian producido variaciones en la concepción teórica que poseía la Unión Soviética de la agresión.

Durante lit reunión del Comité Especial en 1971, Ia cuestión de la intención agresiva continuó a figurar entre los puntos de desacuerdo. Si. para un cierto grupo de $\mathbb{E s}$ stados miembros el elemento intencional era un elemento fundamental en toda definición cle la agresión $n^{43}$, un número no menor de lastados continuaban en la oposición a su: inclusiónit.

Tres tipos de consideraciones aparecen como novedosas en relación a aquéllas expresadas en la reunión de 1970.

La primera está constituida por la aceplación formal de la Unión Soviétioa: de introducir el elemento subjetivo en la definición. Este paso lo ha hecho porque le corresponderitu al Consejo de Seguridad controlar ia veraciclad cle una ausencia de intención agresiva ${ }^{45}$.

La seguncla consideración, más interesante, es aquella que propone distinguir entre intención agresiva y motivas de la agresión. La intención deberá ser entendida como un acto deliberado, lo que permitirá separarla de una agresión ocurrida por accidente o por errorta.

Por último, una consideración de carácter negativa, es aquella expresada por èl Reprentante de Tgipto, en el sentido que introducir la noción de intención agresiva supondria interpretar en forma extensiva las reglas o normas de la Carta de Naciones Unidas. referentes al empleo de la fuerza: ${ }^{ \pm 7}$.

Las próximas reuniones se abren bajo mejores signos, en especial signos cle compromiso. Elio ocurrió en la reunión del Comité Especial, que tuvo lugar en Nueval lork entre el 31 de enero y el 3 de marzo cle 1972.

Si bien no se aicanzó un acuerdo general, es interesante hacer

${ }^{42} \mathrm{Cf}$. Cliaumont (Chr.). La définition de l'agression. op. cil., p; 119;

wEspecialwente Estados Unidos, El Salvador e Italia

"Especialmente Francia, Siria, Colombia, Sudan, Reuador e India.

sVéase las opiniones de Kolesnik 1272 sesión.

"Sesión 1269.

${ }^{47}$ Opiniones del Sr. EI Araby. 
mención de una proposición francesa, que va a constituir el primer esfucrzo del compromiso final.

No obstante, es necesario recordar que para Francia el principio de anterioridad continuaba siendo inconciliable con el criterio de intención, pero dicho país podría considerar a título de compromiso su inclusión. Sin embargo, exigía clos condiciones: una positiva, oträ negativa. La primera condición dice relación a que sería el Consejo de Seguridad a quien corresponderia pronunciarse sobre los motivos perseguidos y las intenciones expresadas por lo Estados parte en una agresión.

Pero de ninguna manera; condición negativa; aceptaba que la falta de intención caracterizada como agresiva de acuerdo a la definición adoptada, pudiera eximir de responsabilidad al Estado que hubiera en primer lugar perpetrado un tombarclco o una invasiónt8.

En el curso de las sesiones de Comité Especial, que tuvieron lugar en Ginebra en 1973, el grupo de trabajo del mismo presentó un texto único que comprendia an preámbulo y siete artículos. Respecto del preámbulo parecería existir ya un gran consenso; diferente era la situación en otros puntos, en especial en relación al Art. 2i del proyecto que trataba de la anterioridad y de la intención agresiva; materias en cloncle existian aún divergencias.

EEsta sesión del lComité fue nuevamente sacudida por la intervención de Francia; que buscó precisar su posición en especial frente al art. 2 del proyecto del grupo de trabajo. Lä delegación francesa no compartía la opinión expresada por algunos representantes en el sentido que no todo empleo de la Iuerza era contrario a la Carta de Naciones Unidas, debiéndose consultar los móviles. Francia era faronable al principio de anterioriclad, pronunciándose en forma positiva por distinguir, por inna parte, la existencia objetiva de un aoto de agresión aplicando la regla o el principio de anterioridad y por otra parte el poder reconocido al Consejo cle Seguridad de volver a examinar dicha calificación (agresión) 40 .

A pesar de las divergencias existentes, la Sexta Comisión de la Asamblea General, consciente que el compromiso definitivo era ya perfectamente posible, decidió convocar nuevamente al Comité Especial, a fin de que pusiera término a su tarea, sometienclo en la próxima sesión de la Asamblea un proyecto definitivo de definición de la agresión. Elio ocurrió el 12 de abril de 1974, fecha en la cual, por consenso, fue aprobada una definición intemacional de la agresión ${ }^{50}$.

"Ce. las expresiones del Sr. Besson en la 1348 sesión.

-Véase la intervención del Representante de Francia en 1441, sesión.

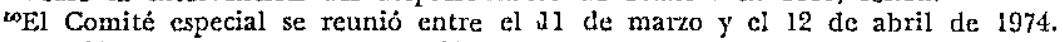
En relación a la forma de aprobación algunas delegaciones fueron decididamente favorables, Francia; otras contrarias, como Israel. 


\section{B. El texto definitivo: un compromiso,} varias incerlidumbres.

Al estudiar el largo proceso de introducción del clemento subjetivo, ros conrespondió observar cómo los Tistados opositores, Unión SoviéLica $y$ Francial especialmente, aceptaron su inclusión en el bien entendiclo que corresponcleria al Consejo de Seguriclad su constatación y análisis.

El compromiso surgío precistmente alrededor de esá idea, materializándose en forma precisa en el artículo e cle la resolución 3314 $\langle\operatorname{xxIx}\rangle^{51}$.

Por la importancia de esta disposición y los posteriores comentarios, crecmos necesario citarla in extenso:

"El primer uso de la fuera atrmada por un Estado, en contravención de la Carta, constituirá prueba prima facie de un acto de agresión, aunque el IConsejo de Seguridad puede concluir de conformidad con la tCarta que la determinación de que se ha cometiclo un acto de agresión, no estaría justificada a la luz de otras circunstancias pertinentes, incluido el hecho de que los actos de que se trata o sus consecuencias no son de sufficiente gravedad".

La simple lectura del articulo 2 nos señala ya su origen: la redacción alambicadic y compleja csconde mal su verclactera naturaleza, la de ser una clisposición de compromiso.

Tanto los partidarios del elemento objetivo de la agresión como aquellos 'que sostenian la importancia del elemento subjetivo, entregaron al Consejo de Seguridad la tarea de solucionar en cacla caso de especie sus pretensioncs teóricas. $A$ ambos el Consejo de Seguridact puede clarles la razón, lo que demuestra el carácter de compromiso que predominó en la redacción de dicha clisposición. El medio por el cual el Consejo de Seguridad puede oscilar entre ambas concepciones está inserto en lá última parte del artículo 2 cuanclo se clice"... a la luz de otras circunstancias pertinentes...".

Así el Consejo de Seguriclad dará la razón a los objetivistas como lo señala pertinentemente |Patrick Rambandby, si se atiene exclusivamente a registrar los hechos materiales, condenando al Estado que primero haya cometido alguno de los hechos cntmerados ejemplarmente en el artículo 3 de la definición. En dicho caso, la simple pre-

s1Nomenclatura definitiva después de aprobación nuevamente por consenso por la Asamblea Gencral.

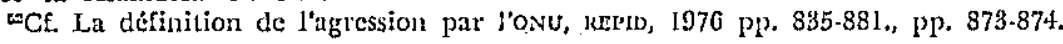


sunción cle agresión que contempla la primera parte del artículo 2 se transforma por la acción del Conscjo, en una certeza.

La situación es diferente si el Consejo de Seguridad decide por el contrario no quedarse en tun simple registro de hechos escudriñando todo aquello que puecla aciarar su resolución, preocupíndose especialmente de la intención agresiva y de la gravedad del acto, respondiendo asi a los intereses y deseos de los subjetivistas.

Si los elementos del compromiso que contenía el artículo 2 dicen relación al sujeto como a su campo de acción, veamos por ahora las principales incertidumbres de clicha disposición... Estas las hemos agrupado en tres: primero, qque debe entenderse por la expresión "otras circunstancias pertinentes", segundo, qué consecuencias tiene un no pronunciamien to del Consejo de Seguridad y, por último, quién puede utilizar la frase en que se hace referencia a "violación de la Carta".

Después clel acercamicnto que existió entre la Unión Soviética $y$ los paises occidentales a propósito de la inclusión de la intención agresiva, se sucedieron especialmente en la última sesión del Comité Especial, una serie de declaraciones interpretativas por parte de las Grancles :Potencias en el sentido de incluir el elemento subjetivo en la expresión "otras circunstancias pertinentes". Ante tales declaraciones, varios paises del Tercer Munclo protestaron, especiaimente Argelia ${ }^{53}$.

El segundo aspecto se refiere a la situación que ocurriria si no obstante la realización, de algunos de los hechos enumerados en el artículo 3, el Consejo de Seguririclad no se pronunciara. Este punto ha siclo tratado directamente por el profesor Zourek $y^{\prime}$ en forma tangencial por Ramband.

El ex-presidente cle la Comisión de Derecho Internacional de Naciones Unidas, comentando la expresión "otras circunstancias pertinentes", estima que el Consejo de Seguridad no podía hacer abstracción de la pruéba (desconocer el principio de anterioridad), sino por medio de una votación en conformidad a las reglas de procedimiento vigentes y mientras clla no se c[cctúe, la pruaba de la agresión permanece $y$ produce sus efectos ${ }^{\text {trt }}$. (Desgraciadamente no podemos seguir en este punto all profesor Zourek. Según la Carta de Naciones Unidas, como bien lo expresa Ramband ${ }^{5 \sigma}$, no existe una agresión por sí misma, siendo necesario un juicio motivado del Consejo cle Seguridacl. Esta consintación aparece indirectamente confirmada si se analizan conjuntamente los artículos 2 y it de la resolución $3314\langle\operatorname{xxix}\rangle$.

Mediante el artículo 4 del Consejo de Seguridad puede completar

"Véanse las declaraciones del seĩor Boulbina $y$ la de los representantes de Cuba y Egipto en $1 \mathfrak{a} 1479$ y 1483 sesión, respectivamente.

* Enfin une définition de l'agression... op. cit., p. 22: 
de su propia iniciativa, los casos contemplados en el artículo 3. Por el contrario, y según el artículo 2, el Consejo de Seguridad puede descalificar como acto de agresión, algún hecho de los contemplados en el antículo 3, y ello por motivos que él sólo aprecia.

El hecho agresivo complementado con el principio de anterioridad es una presunción de una posible agresión, pero ésta será calificada después del juicio soberano del Consejo de Seguridad. No existe agresión sin calificación.

EEl último punto de incertidumbre en la redacción del artículo 2, se réfiere a la expresión "violación de Ia ICarta". Para Francia especialmente esta reférencia sólo puede ser utilizada por el cConsejo de Seguridad y de ninguna manera por el Estado agresor, quien no puede pretender haber actuado conforme a su propia interpretación. ${ }^{56}$. WEs decir, se rechaza de plano la idea: que corresponde probar a la víctima que se ha empleado la fuerza en violación de la Carta de Naciones Unidas.

\section{CONCLUSIONES}

A modo de conclusión, quisiéramos referirnos brevemente al objetivo principal de nuestro estudio, es decir al lugar que ocupan los elementos objetivos y subjetivos en la definición de la agresión, pero esta vez buscando algún criterio que permita sobrepasar esta querella, hasta el momento muy estéril.

Patrick Ramband, en su artículo ya citado, nos ofrece uno: el criterio de la gravedad. Según este autor existiría una sola forma de aprehender de "manera objetiva" el elemento subjetivo, la intención agresiva. Ella se encontraria en el propio artículo 2, al disponer que se considerarían también si los actos o sus consecuencias no han sido de una gravedad suficiente. El criterio de gravedad del hecho como de sus consecuencias nos permitiría distinguir un simple incidente de fronteras, aun cuando ello implicara primera intervénción e invasión de tropas, de una a'gresión verdadera.

El criterio de gravedad propuesto deberia además ser apreciado en función de la amenaza que los hechos del caso en cuestión, significan para el Estado víctima.

Un elemento de mayor contenido objetivo como la gravedad podría tranquilizar a Jos partidarios de una como de lạ otra concepción; y serviria como línea clirectiva, más real e imparcial, para el Consejo de Seguridad.

${ }^{\star}$ Cf. La définition de l'agressión par l'oNv, op. cit., p. 877.

${ }^{\circ} \mathrm{Cf}$. las opiniones del señor Bessou en la 1474 sesión. Iguales comentarios porparte de Rumania; Yugoslavia y Argelia. 